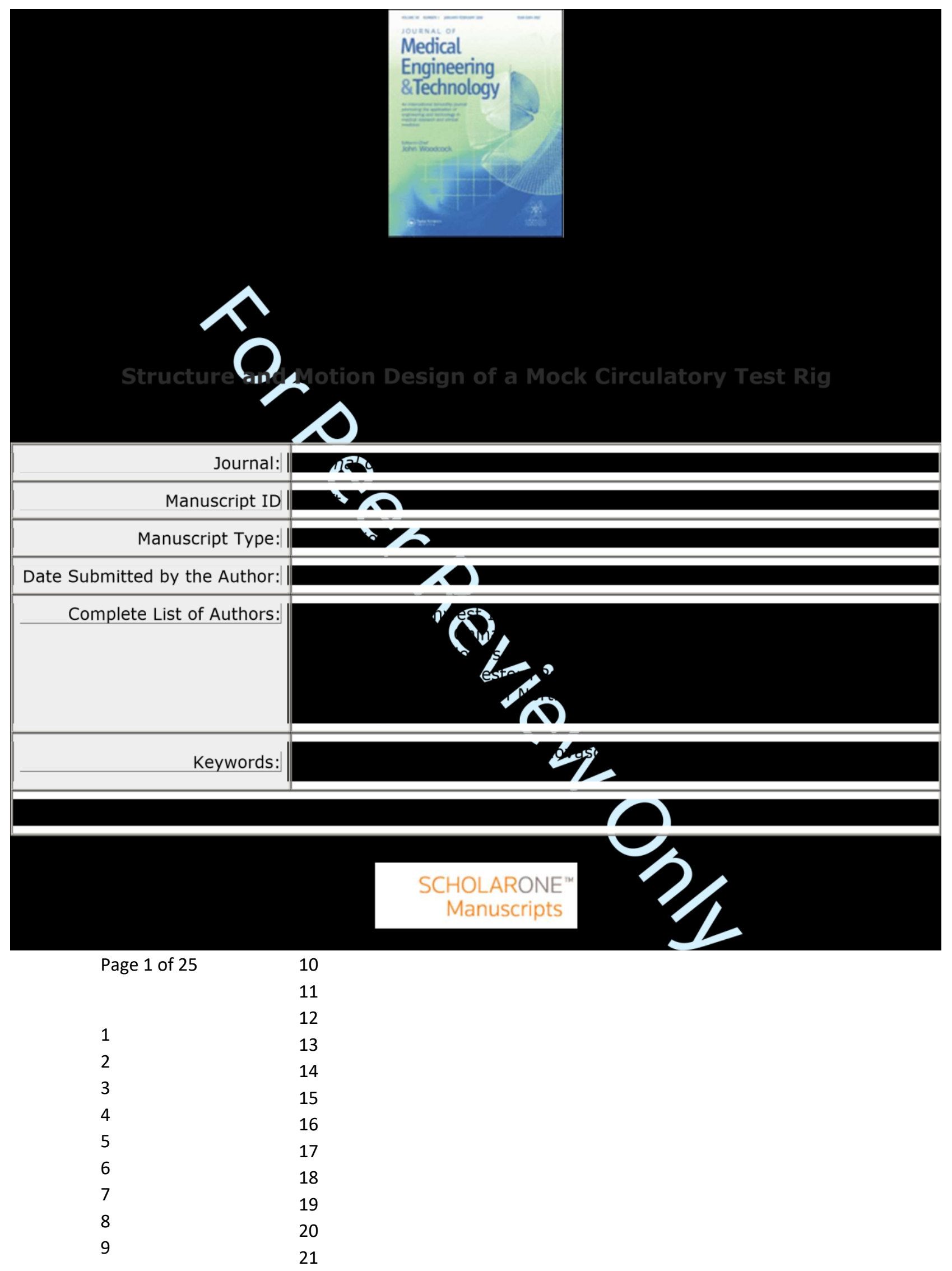




\section{Structure and Motion Design of a Mock Circulatory Test Rig}

Yuhui Shi *, Theodosios Korakianitis \$, Zhongjian Li \&, and Yubing Shi

\#

* Northwest Institute of Mechanical and Electrical Engineering, Shaanxi Province,

China

\$ Parks College of Engineering, Aviation and Technology, Saint Louis University,

\& College of Automation, Northwestern Polytechnical University, China

\# Faculty of Arts, Science and Technology, University of Northampton, Northampton,

\section{Corresponding author:}

Faculty of Arts, Science and Technology, University of Northampton,

University Drive, Northampton, NN1 5PH, UK

Email: yubing.shi@northampton.ac.uk

The total number of words in the main text:4541

USA 
$U K$

Yubing The number of words in the abstract: 158

Shi

The number of figures: 7

The number of tables: 2

Page 2 of 25

\title{
Structure and Motion Design of a Mock Circulatory Test Rig
}

9

\begin{abstract}
Mock circulatory test rig (MCTR) is the essential and indispensable facility in the cardiovascular in vitro studies. The system configuration and the motion 14 profile of the MCTR design directly influence the validity, precision, and accuracy of the experimental data collected. Previous studies gave the 17
\end{abstract}


schematic but never describes the structure and motion design details of the MCTRs used, which makes comparison of the experimental data reported by 20

different research groups plausible but not fully convincing. This paper presents the detailed structure and motion design of a sophisticated MCTR 23 system, and examines the important issues such as the determination of the ventricular motion waveform, modelling of the physiological impedance, etc.

in the MCTR designing. The study demonstrates the overall design procedures from the system conception, cardiac model devising, motion planning, to the motor and accessories selection. This can be used as a reference to aid researchers in the design and construction of their own in-house MCTRs for cardiovascular studies.

Keywords: mock circulatory test rig; cardiovascular dynamics; in vitro test, 36 structure design; motion design

\section{Introduction}

Clinical investigations play an important role in the cardiovascular physiology 43 research. However, due to various limitations (e.g., the complex geometries of the

cardiovascular organs, the difficulty in isolating the influence from each of the individual physiological factors, and the size of the sensors etc.), not all physiological and pathological phenomena can be observed directly with clinical studies on the human body (1). Thus it is necessary to construct artificial systems to simulate the functioning of the human circulatory system, so that in vitro experiments can be 


$15 \quad 49$

$16 \quad 50$

50

carried out on the artificial systems instead. Such an artificial system is often implemented as a mechanical-electrical-hydraulic assembly and is tuned to produce different blood flow conditions corresponding to the healthy and diseased status in the human body (2). The mechanical-electrical-hydraulic system is usually called a mock circulatory test rig (MCTR). Past designs of MCTRs simulate steady or pulsatile flow, depending on the goals of the experiments. Using these MCTRs, researchers gained more insight into not only the blood flow physics (i.e., changes of overall flow field structure as well as the variations of velocity, pressure, shear stresses, and the different forces acting on the native organs) (2-12), but also detailed information for the design and performance analysis of prosthetic devices 
such as

artificial

heart

valves,

stents, and

ventricular

assist

devices

$(1,2,12-$

38). The

MCTR

has

become a

fundament al and indispensable facility in the in vitro study of the cardiovascular physiology.

The system configuration and the motion profile of the MCTR design determine to what degree the simulated cardiac flow mimics the physiological reality, and thus directly influence the validity, precision, and accuracy of the experimental data collected in the in vitro studies $(1,2)$.

Given the importance of the MCTR design, it is surprising that no structure and motion design details of the MCTRs have ever been reported in the open literature so far, and those published cardiovascular in vitro studies usually described only the schematic of the MCTR followed by a brief list of the components used in the system. Lacking of such technical details, comparing the experimental data reported by different research groups becomes plausible but not fully convincing. Besides, researchers who plan to build their in-house MCTRs but have less design experience would find themselves helpless after a fruitless literature survey. To fill the gap, this paper presents the detailed structure and motion design of a sophisticated MCTR

system, which can be used as a reference by cardiovascular researchers in their planning to build in-house MCTRs. Also it is hoped that this paper would initiate further in-depth discussions among researchers about the improvement of the MCTR designs, which as a result contributes to the continuous advances and refinement of 
the MCTR technology to support the ever-increasing requirements about the reliability and the accuracy in the cardiovascular in vitro studies.

\section{System Design}

The aim of the current MCTR design is to provide an embodiment of a fundamental electric-mechanical platform that simulates the hydro-dynamic characteristics of the 23 human circulatory system, to facilitate the in vitro measurement of blood flow features and testing of response dynamics in cardiovascular prosthetic organs.

Besides, the MCTR design should be able to simulate the circulatory response in a range of healthy and diseased conditions: from the healthy rest condition to the exercise condition, and even the heart failure condition. For such purposes, the operating parameters for the MCTR are first chosen as shown in Table 1, by referring 36 to the ranges of the physiological variables reported in the literature (39-41).

(Table 1 comes here)

\subsection{System configuration}


The MCTR design comprises two circulation loops: the systemic loop and the pulmonary loop. In each loop, two throttle valves and a compliance unit are used to simulate the corresponding physiological impedance, and a tank separated from the rest of the system by a one-way check valve is used to provide the correct mass of fluid in circulation (adjusted during system commissioning). Three pressure transducers and two flow transducers are installed in each loop, to measure the 26

27

28

29

30

31

32

33

34

35

36

37

38

39

40

41

42

43

44

45

46

47

48

49

50

pressure in the atrium, the ventricle and the main artery, and the flow rates in the artery and the vein. Fig. 1 shows the system configuration of the MCTR design. 


\section{2}

Structure

Design of the heart model

The heart model is the central part in in the MCTR design. The heart design consists mainly of five parts, as illustrated in Fig. 2.

Except the difference in shape in the left and right ventricles, the whole heart model is almost symmetrical along the centre plane. As shown in Fig. 2, parts A, B and E represent the ventricles and the atriums. The ventricle part is separated into Part A and $\mathrm{B}$ in order to minimize the difficulty in manufacturing the ventricle chambers. Parts $\mathrm{D}_{\mathrm{KG}}$ and $\mathrm{D}_{\mathrm{v}}$ are two silicon rubber membranes that represent the atriumventricle septum and the ventricle wall, and they are separated by Part $\mathrm{C}$ to eliminate the potential motion interference between them. Besides these main parts, some accessories such as heart valves, sealing components and motion mechanism etc. are used to help mimicking the proper function of the model. Under the action of the external motion control system, blood analogue is circulated in the heart model to simulate the functioning of the heart. The arrows in the figure show the flow direction of the blood analogue.

In the figure, LA is the abbreviation of left atrium, LV for left ventricle, RA for right atrium, RV for right ventricle, mi for mitral valve, ao for aortic valve, ti for tricuspid valve and po for pulmonary aorta.

In Parts $\mathrm{A}$ and $\mathrm{B}$, the left and right ventricle chambers covered under the ventricle sac 
Dv are

filled with

water.

Water is

filled and

drained

through

flow

channels

near the 
apex of the ventricle chambers. The flow channel and the ventricle chambers are connected through honey combs that are tiled towards the ventricle bottom direction, in order to eliminate the possible water jet effect acted on the ventricle sac $D_{v}$ when

water is filling in the chambers to simulate the systolic phase.

Fig. 3 shows the MRI measured geometry of the left and right ventricles at the instances of peak-systole and end-diastole in a heart cycle, and Fig. 4 shows the corresponding ventricular volume change in the left ventricle, which have been reported in a previous study (42). The MCTR design aims to give realistic description 20 of the cardiac motion and functioning, but does not need to duplicate every anatomical details. The irregular shape of the right ventricular is very difficult to 24 fabricate mechanically, thus it is represented using the same geometry as that for the left ventricular, but working in the pressure range compatible with the pulmonary circulation. In the MCTR design, the ventricle sac $\mathrm{D}_{\mathrm{v}}$ in the unstressed condition exhibits the ventricle shape in the peak-systole in the rest condition, and the internal 33 wall of Part A and B are cut into the ventricle shapes in the end-diastole in the extreme exercise condition. When the amount of the water in the space between the 37 
Page 7 of 25

19

20

21

22

23

24

25

26

27

28

29

30

31

32

33

34

35

36 37 36
Part $D_{\text {К̆ }}$ and Part E combined to represent the blood flow in the left and right atriums,

8

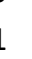

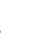

(1)

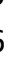
27
28 9 2

ventricle sac and the internal wall of Part A and B is changed using the ventricular motion control motor and piston, blood analogue flows in and out of the space LV and $\mathrm{RV}$ to compensate the volume change induced by the water flow, thus mimicking the entricular volume changes as shown in Fig. 4. The LA and RA chambers are epresented with cylindrical shaped spaces whose volumes approximately equal the MRI measured left and right atrial volumes.

(Figures 3 and 4 come here) 
1

2

3

4

5

6

7

38

39

40

41

42

43

44

45

46

47

48

49

50

the aorta

and the

pulmonary

artery.

Two heart

valves are

equipped

on the

silicon

septum

51

52

53

54

55

56

57

5

8

5

9
$D_{\mathrm{KG}}$, and they are functioned as the mitral valve and the tricuspid valve in the heart. The two valves and the septum are attached to and driven by the motion control mechanism which moves in the vertical direction, thus to simulate the bulging of the septum in the heart in the diastolic phase. Two vertical channels in Part E represent the roots of the aorta and the pulmonary artery. Two more heart valves are installed near the inlet to the aorta and the pulmonary artery to simulate the aortic valve and the pulmonary valve.

\subsection{Motion design for the system}

(Figures 5 and 6 come here)

Proper functioning of the heart model depends largely on the careful motion planning and synchronisation of the motion control system in the septum driving mechanism and the ventricle driving mechanism. Fig. 4 above shows the typical left ventricular volume change in a heart cycle measured with MRI, which is used as the blueprint in the motion profile design of the ventricular driving mechanism in the MCTR design. To reduce the impact and vibrations in the driving motor and the 
mechanica

1 parts in

the

MCTR,

the

volume

change in

Fig. 4 is

further

approxima

ted with

piece-wise

harmonic waveforms, as exemplified in Fig. 5, which is represented by the following equation:
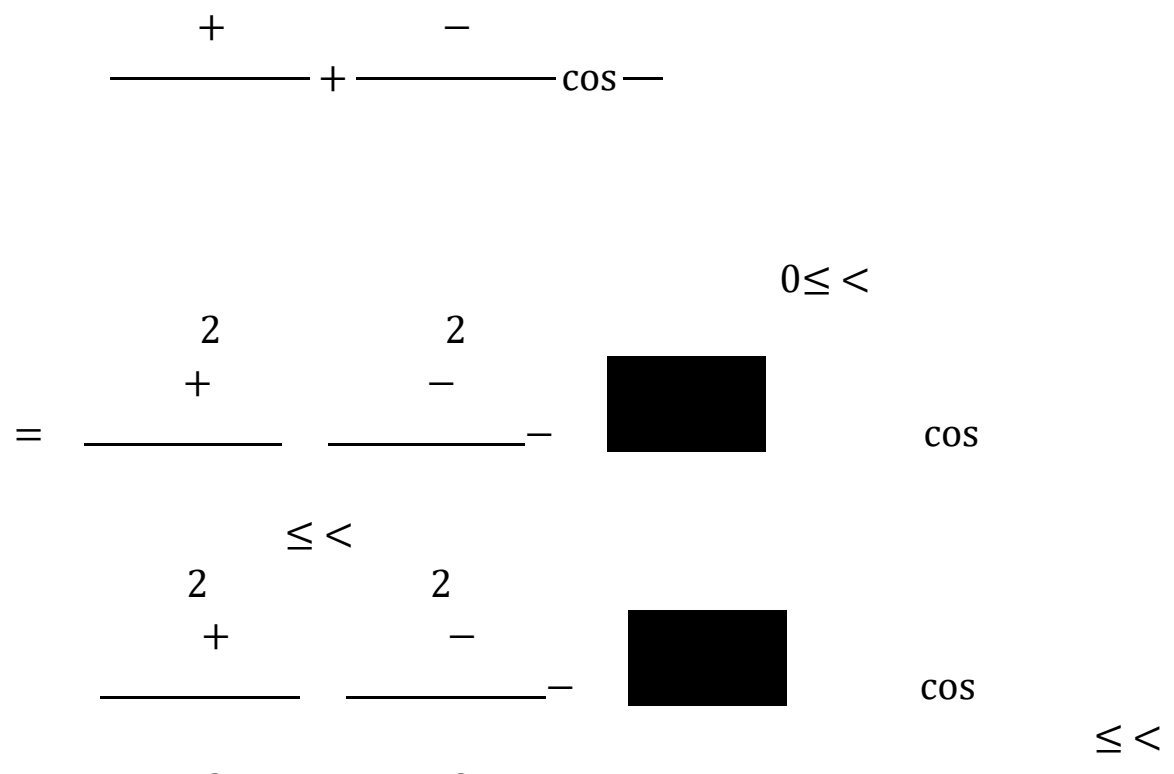

Equation (1)

In the equation, $\quad, \quad$, and are the maximum, the minimum, and the saddle

point values in the volume change curve; and are the time corresponding to the transition points in the waveform segments, and is the heart period. As can be observed in Fig. 5, the harmonic waveforms shown is based on a set of example

values for $\mathrm{V}$ and $\mathrm{T}$, and they can be easily adapted to other suitable values to describe 
the patient-specific and disease-specific conditions which the researcher expect to simulate.

For both the left and the right ventricles, a piston actuated by a linear motor is used to

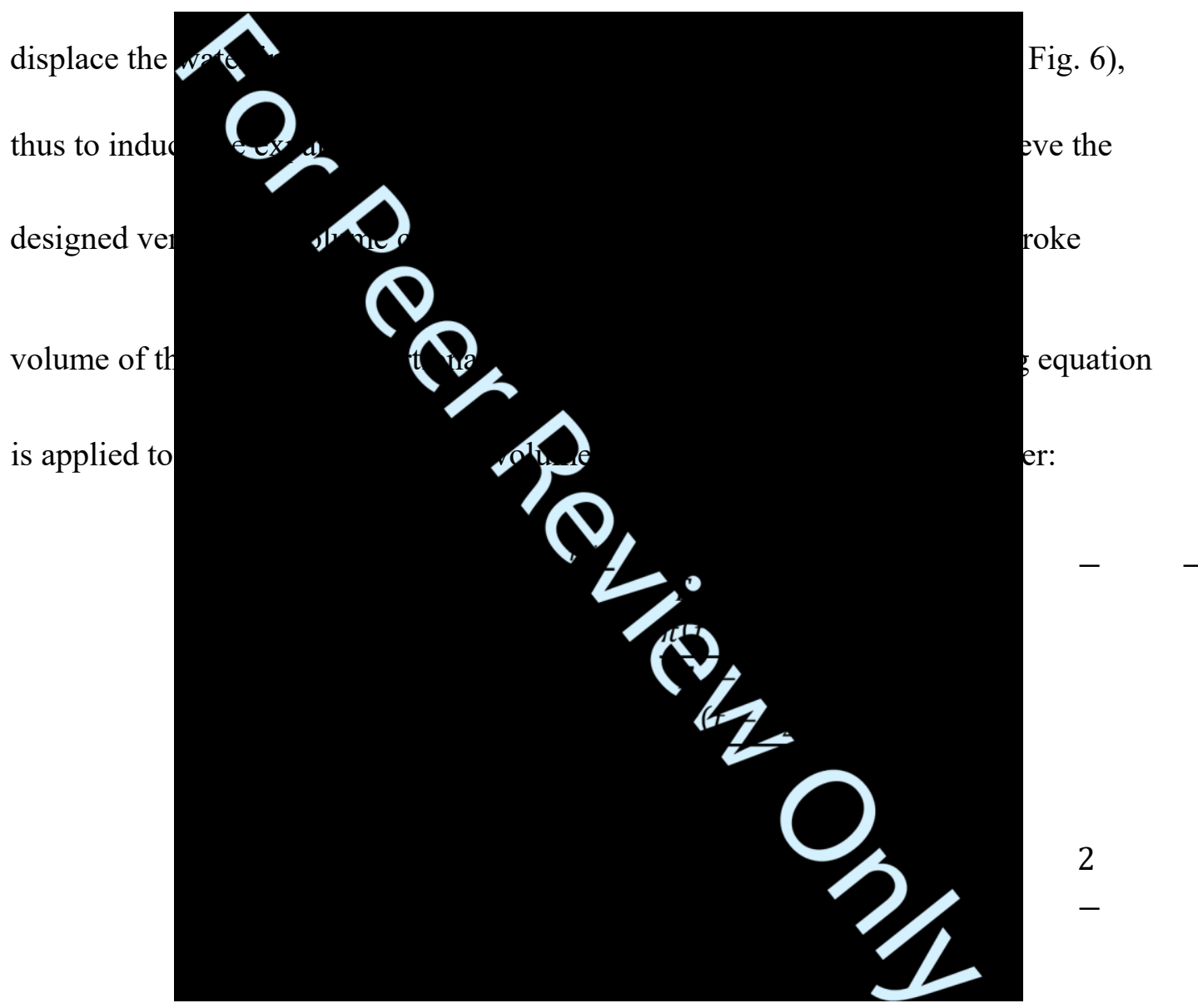




$$
\begin{array}{llll}
= & + & \cos & \\
& & \\
< & & & \\
2 & 2 & & \\
+ & - & & \\
2 & + & 2 & \cos \\
& & & \\
& & \leq<
\end{array}
$$

Equation (2)

In the equation, , , and are the maximum, the minimum, and the saddle point values in the stroke profile curve; , and are the same as that in equation (1). Considering the dimensions of the left and right ventricles measured in the MRI, 45

46 in the current MCTR design the diameter of the piston head is chosen as $50 \mathrm{~mm}$. To 47

While to produce the stroke volume of about $110 \mathrm{ml} /$ beat $(160 \mathrm{ml} /$ beat $)$ in the extreme 
49

50

exercise condition (39), the piston should have a stroke of:

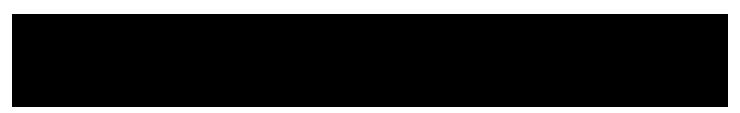

The working stroke of the piston can be chosen as $0.08+$. To leave enough margins, the overall stroke of the piston is chosen as $0.1+$, which can be used in the selection of the linear motor. For specific modelling cases, the stroke value can be adapted to

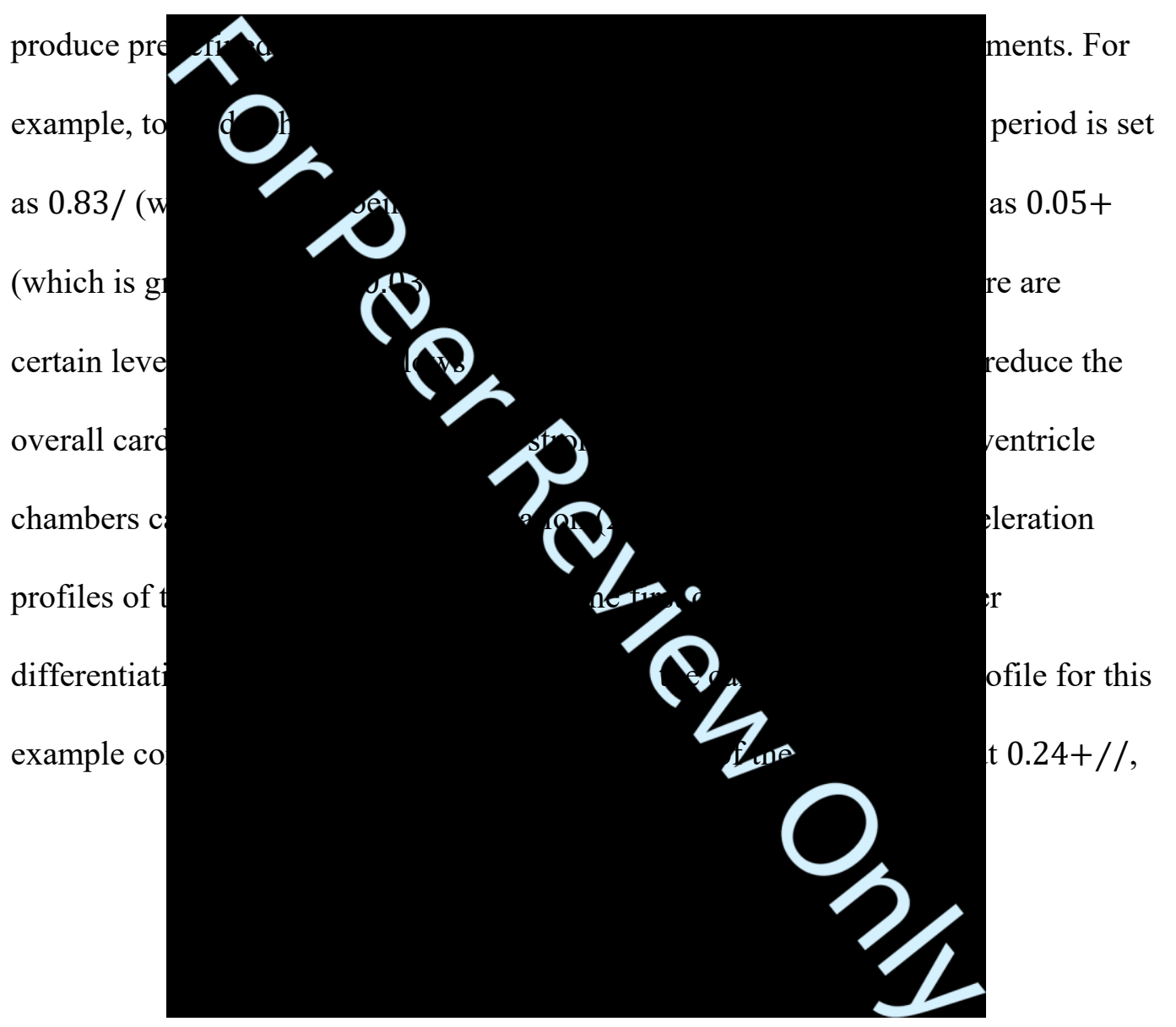


and the

maximum

acceleratio

$\mathrm{n}$ is

$3.2+/ /$.

The

motion

profile for

the

exercise condition have the same shape as illustrated above but elevated stroke, velocity and acceleration values but reduced heart period value, due to the increased cardiac contractility and heart rate in the exercise condition. For the extreme exercise condition of $180012 /+34 /$ for the heart rate and $160+5012 /$ for the stroke volume (39), using the piston stroke of $0.1+$ calculated above, the maximum velocity of the piston is about $2.41+/ /$, and the maximum acceleration is $132+/ /$. While to simulate the heart failure condition, the piston stroke needs to be reduced and the heart rate increased. The detailed motion profiles for the stroke, velocity and acceleration can be calculated in the same way as

above.

(Figure 7 comes here)

The atrial-ventricular septum moves in a heart cycle due to the contraction of the heart muscle, which is in phase with the ventricular motion but with a reduced magnitude, 11 thus the septum actuating mechanism has the same motion trajectory as the piston for the ventricle. After checking the MRI measured data, stroke for the septum driving mechanism is set as $25++$, which produces a maximum velocity is $0.496+/ /$ and a 
maximum acceleration of

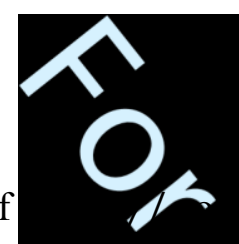

in a healthy

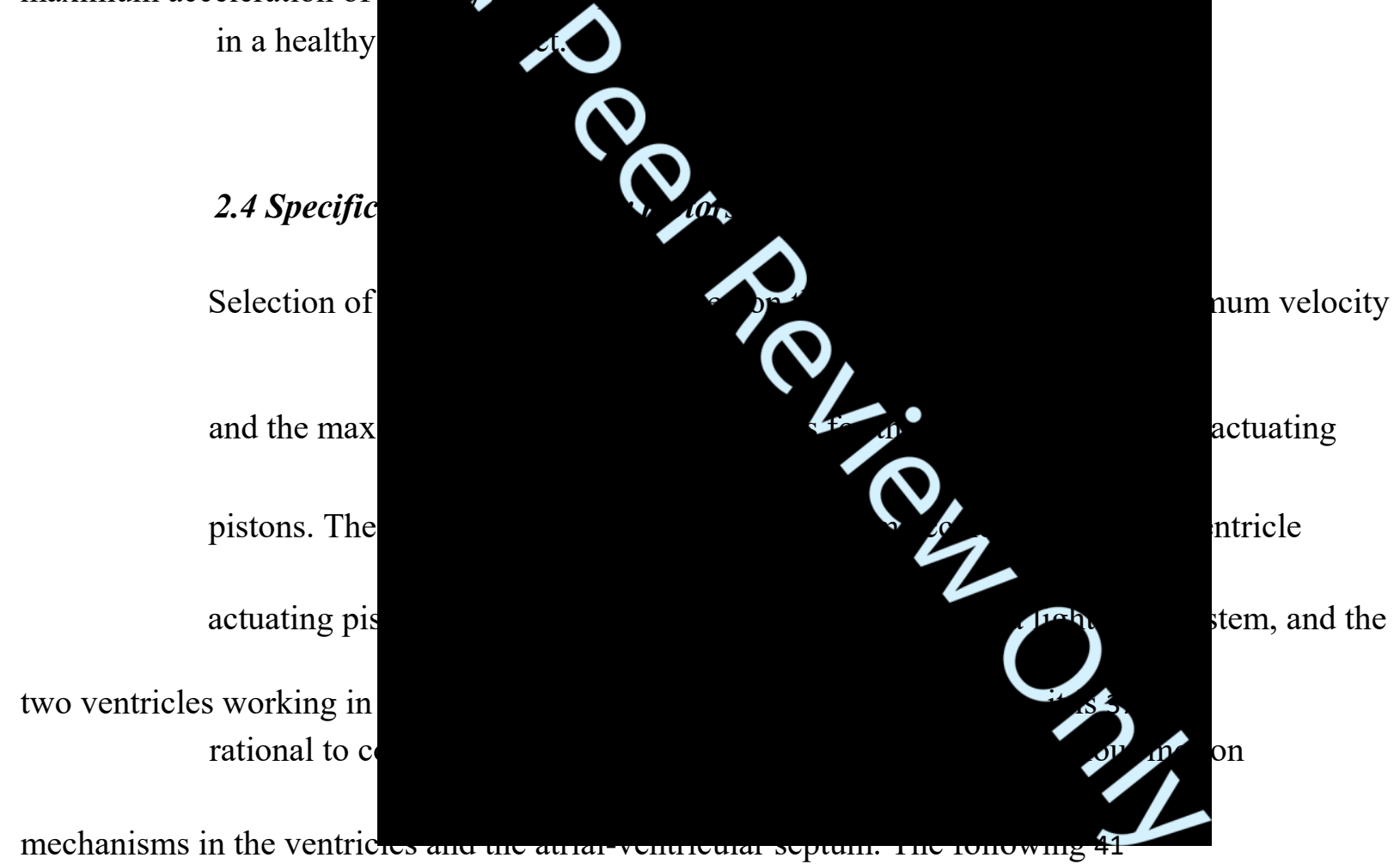

evaluation of the force requirement is based on the left ventricle driving motor.

The piston driving force is a summation of the pressure force, the inertial force due to fluid acceleration, and the frictional force. To calculate the pressure force, the peak pressure in the left ventricular chamber is set as $200++67$ (which gives sufficient 
margin over the upper limit of $160++67$ for the hypertension condition (43)), and the pressure force is estimated as:

$$
F P A_{p}=\quad=13.610 \times \quad{ }^{3} \times 9.8 \times 0.2 \times 0.25 \pi \times 0.05^{2}=52.34(N)
$$

The inertial force exists in the piston and the circulating fluid in the heart chambers

and the flow channel between the aortic valve and the compliance element, with the

piston plays the major part due to the greater density of the steel piston material 11

compared with the density of the blood analogue. Assuming the piston has a diameter

of $50++$ and a thickness of $10++$, while the piston rod has a diameter of $10++15$

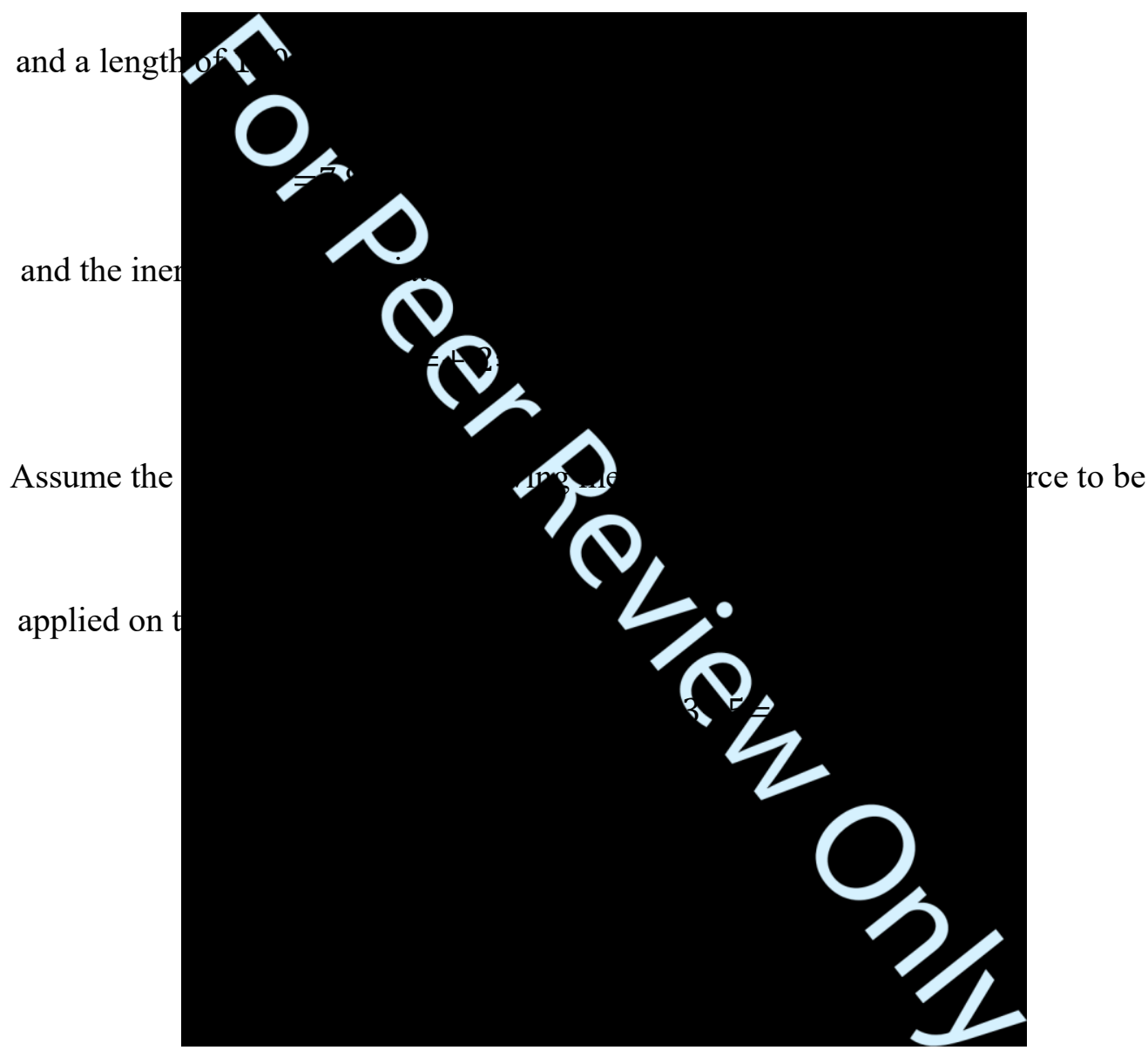


1

4

5

6

7

32

33

34

$36_{37}$

38

39

40

41

42

43
45
46

47

48

49

50

catalogue, the force output from the piston is selected as $200<$. The right ventricle 35

has a smaller pressure level thus the force requirement is also smaller. The atrial

septum driving piston not only works under lower pressure but also experiences

smaller acceleration. Since $200<$ is not a big value, it is convenient to use the same

Use a safety factor of $2 \sim 3$, and round the force to a nominal value in the product

pecifications for all the four ventricle and atrium driving pistons. The specifications 44

for the linear motors to drive the ventricle/atrium pistons are then summarised and

listed in Table 2.

(Table 2 comes here)

\subsection{System accessory}

Besides the main parts of the heart model, the piston and the driving motor, some

Page 12 of 25 
accessories are needed to form the complete MCTR system as detailed in the following.

\subsubsection{Valve and compliance units, to simulate the physiological impedance}

Sharp et. al. (2) analysed the characteristics of the physiological impedance in the artery network, and suggested that the three-element Windkessel model is the simplest

model that could describe both the low frequency and high frequency features of the physiological impedance, while the two-element Windkessel model as used in majority of the previous MCTR designs was unable to reveal the high frequency

features. Thus the three-element Windkessel model is used in both the systemic and 23 the pulmonary loops in the current MCTR design. For this purpose, two throttle

valves are used to function as the characteristic resistance and the peripheral 27 resistance, and a compliance unit to simulate the elastic effect of the artery. Generally, the overall resistance value produced by the two resistances equals to the ratio of the mean pressure to the mean flow rate in the loop, and the characteristic resistance is about one tenth of the peripheral resistance. Besides, the human aorta has a diameter 36 of about $25++$, thus to have better geometrical resemblance and to avoid the unnecessary pressure loss and flow disturbance, the internal diameter of the throttle 40 $25++$. The compliance unit is made of an elastic rubber sac $42 \quad$ valves is chosen to be emerged in a container filled with compressed air, so that different values of air 
pressure result in different values of the compliance. During system commissioning, the air pressure in the compliance and the openings of the valves are continuously 49 adjusted, until the pressure and flow responses in the system match that in the physiological conditions as reported in the literature.

Page 13 of 25

\subsubsection{Water tank, to provide the necessary flow reserve 4}

A normal adult of $75 \mathrm{~kg}$ body weight has about 5 of blood in the circulation system

$(40,41)$. In the MCTR design, two water tanks, each has an overall volume of 4 and contains 3 of blood analogue, are used to represent the blood storage capacity in the

12 systemic and the pulmonary veins. The overall volume of the blood analogue exceeds 13 14 that of 5 in the human body but this does not influence the system response. The 15 water tanks can be placed on the same horizontal level with the other system parts, to

simplify the system settlement; or they can be placed on shelves so that they are on a higher altitude to produce the right level of the preload (about $10++67$ in the 
normal condition) to the left and right atriums.

\subsubsection{Silicon rubber tube, to connect different parts in the system}

Silicon rubber tube provides a flexible but water-tight connection of the components

in the MCTR design. To be compatible with the geometry of the artery, the silicon 32

rubber tube also has an internal diameter of $25++$.

\subsubsection{Blood analogue}

To match the density of $1060: 7+/{ }^{9}$ and the viscosity of 3.2?@ in the real human blood $(40,41)$, a mixture of glycerine and water is used as the working fluid. If the

MCTR is to be used for applications involving sophisticated optical observations of 45

the velocity distributions, such as in the Particle Image Velocimetry measurement of

the flow field, the test section often needs to be manufactured using transparent 49

materials (e.g., Perspex), which has a different refractive index to the blood analogue and may cause optical distortion in the images captured. In such situations, chemicals such as sodium iodide $(<2 \mathrm{~A})$ can be added to the blood analogue so that the refractive

index of the fluid matches the index for the transparent wall material in the observation windows, to eliminate the potential of optical distortion. However, this 
will change the density and the viscosity of the final working fluid and a compromise among the different aspects of the fluid properties needs to be considered.

\subsubsection{Pressure and flow transducers}

Three pressure transducers and two flow-rate transducers are installed in each loop, to measure the pressure in the atrium, the ventricle and the main artery, and the flow rates in the artery and the vein. The operating range of the pressure transducers is chosen as $-20 \sim 300++67$, in case there may be temporary pressure overload in the system. Electromagnetic type or ultrasound type flow transducers are good candidates

for the measurement of the pulsatile flow in the systematic artery and the pulmonary 27

$$
\begin{aligned}
& \text { / and it may } \\
& \text { artery positions. Considering that the } \\
& \text { normal cardiac output is } 5+34
\end{aligned}
$$

raise to about $28+34$ / in the maximum exercise condition (39), the operating range

of the flow transducer can be set as $-10 \sim 30+34 /$. Flows in the simulated systematic and pulmonary vein positions are much steady, so the rotameters can be 
used in these locations to save the expense.

\subsubsection{Computer monitoring and control system}

A computer monitoring and control system is needed to record the pressure and flow response in the MCTR, as well as to command the linear motors to generate the necessary designed motions in the ventricle/atrium actuating pistons. A normal PC enhanced with the hardware (suitable A/D \& D/A board) and the software (accompanying data acquisition and motion control program) support is OK for the purpose. Besides, the pressure and flow signals from the transducers are weak signals, and need to be conditioned and amplified before being picked up by the data

acquisition system, thus the signal conditioning and amplifying circuits accompanying the transducers need to be purchased. The motion command signals sent by the 6 computer to the linear motors often need to be amplified with a separate amplifier as well. With the computer monitoring and control system, signals from the pressure and flow transducers and from the motor driving channels can be displayed real-time on the computer screen or saved as data files for further post-processing. 


\subsubsection{Other application specific components}

The MCTR system provides the fundamental platform for the in vitro study, while for specific applications it often needs to be adapted or expanded to include other 23

measurement components. For example, to observe the flow field in a new artificial

heart design or in a patient-specific vessel segment geometry, the artificial heart or the 27

vessel segment can be fabricated using transparent materials and then embedded in the MCTR for flow measurement.

\section{Discussion}

MCTR is widely used in the in vitro study of the cardiovascular physiology. Plenty of

MCTRs have been built in the past. Although the general configurations of these 44

MCTRs were introduced in the literature, the structure and motion design details have 

previous MCTR designs, the current design adopts improved

seldom been explained. This paper presents a MCTR design with sophisticated

structures and describes the technical details that were missing in the literature, thus

provides a reference to researchers who are planning to build their own MCTRs. Compared with the 
cardiac and vascular system models, thus can provide more in-depth knowledge about the cardiac dynamics and vascular hemodynamics to the researchers. The proposed MCTR design when fully implemented can be used as a platform to aid the clinical study of the native cardiovascular system in healthy and diseased conditions and the performance evaluation of prosthetic cardiovascular devices.

The proposed MCTR design is focused on the real-size modelling of the circulatory system. It is possible that in some situations scaled-up modelling is need to facilitate the observation of the flow field details in some confined regions of the cardiovascular flow. In such cases the dimensional analysis can be applied to change the geometrical dimensions of the cardiac/vascular model and the motion parameters 24 (velocity, frequency etc.), and to maintain the non-dimensional numbers (e.g.,

Reynolds' number, Womersley number) so that the dynamic similarity between the MCTR model system and the original physiological system is guaranteed. Once the revised geometrical dimensions and the notion parameters are decided, the same 33 procedures as presented in this paper can be applied to determine the design parameters for the actuating pistons and the driving linear motors, thus to finalise the 
MCTR design.

The study presents a sample MCTR design, elaborates on the different aspects of the structure and motion design considerations, and demonstrates the procedures for determining the geometrical parameters and the motion profiles. Researchers can use this as the reference and adapt the design by revising the geometrical and kinematic parameters, removing and incorporating system components, to suit different research

needs. For example, if the motion of the atrial-ventricular septum is not needed then 53 the related parts can be moved, and this helps to further simplify the system design

5 then the stroke, velocity, and acceleration requirements of the driving mechanism can 6 all be lowered so that cheaper motors can be used; if the pulmonary loop is not relevant then only half of the current MCTR design can suffice the research needs.

A deficiency of the current study is that the designed MCTR has not been physically

built, thus no pressure and flow response can be demonstrated for comparison with the typical response curves reported in the physiological textbooks for validation

purpose. This will be conducted in the next stage of the study once the necessary 20 resources are secured. 
27 This paper presents the detailed structure and motion design of a sophisticated MCTR 28

designing. The study demonstrates the overall design procedures from the system conception, cardiac model devising, motion planning, to the motor and accessories selection. This can be used as a reference to aid researchers in the design and 39 construction of their own in-house MCTRs for cardiovascular studies.

\section{Conflicts of interest}

None declared.

\section{References}

1. Leefe SE, Gentle CR. A review of the in vitro evaluation of conduit-mounted cardiac valve prostheses. Med Eng Phys. 1995 Oct;17(7):497-506.

2. Sharp MK, Dharmalingham RK. Development of a hydraulic model of the human systemic circulation. ASAIO J. 1999 Dec;45(6):535-40. 
3. Boes S, Ochsner G, Amacher R, Petrou A, Meboldt M, Daners MS. Control of the Fluid Viscosity in a Mock Circulation. Artif Organs. 2018 Jan;42(1):68-77.

4. Heise M, Krüger U, Rückert R, Pfitzman R, Neuhaus P, Settmacher U. Correlation of Intimal Hyperplasia Development and Shear Stress Distribution at the Distal End-side-anastomosis, in vitro Study Using Particle Image Velocimetry. Eur J Vasc Endovasc Surg. 2003 Oct 1;26(4):357-66.

115 5. Kaufmann TAS, Wong KC, Schmitz-Rode T, Steinseifer U. Mimicking of 12 Cerebral Autoregulation by Flow-Dependent Cerebrovascular Resistance: A

Reliability of thermodilution derived cardiac output with different operator 22 characteristics. J Clin Monit Comput. 2018 Apr 1;32(2):227-34.

23

$24 \quad$ 8. Misgeld BJE, Werner J, Hexamer M. Robust and self-tuning blood flow control 25 during extracorporeal circulation in the presence of system parameter 26 uncertainties. Med Biol Eng Comput. 2005 Oct 1;43(5):589-98.

27
9. Ninomiya S, Tokaji M, Tokumine A, Kurosaki T. Virtual patient simulator for the perfusion resource management drill. J Extra Corpor Technol. 2009 Dec;41(4):206-12.

10. Piatti F, Palumbo MC, Consolo F, Pluchinotta F, Greiser A, Sturla F, et al. Experimental quantification of the fluid dynamics in blood-processing devices through 4D-flow imaging: A pilot study on a real oxygenator/heat-exchanger module. J Biomech. 2018 Feb 8;68:14-23.

11. Seong J, Jeong W, Smith N, Towner RA. Hemodynamic effects of long-term 
13. Brücker C. Dual-camera DPIV for flow studies past artificial heart valves. Exp Fluids. 1997 Apr 1;22(6):496-506.

14. Crosby JR, DeCook KJ, Tran PL, Smith RG, Larson DF, Khalpey ZI, et al. Physiological characterization of the SynCardia total artificial heart in a mock circulation system. ASAIO J Am Soc Artif Intern Organs 1992. 2015 Jun;61(3):274-81.

15. Crosby JR, DeCook KJ, Tran PL, Betterton E, Smith RG, Larson DF, et al. A Physical Heart Failure Simulation System Utilizing the Total Artificial Heart and Modified Donovan Mock Circulation. Artif Organs. 2017 Jul;41(7):E52-65.

Page 19 of 25

5

17. Feng Z, Umezu M, Fujimoto T, Tsukahara T, Nurishi M, Kawaguchi

D. In vitro 8

hydrodynamic characteristics among three bileaflet valves in the mitral position.

9

\begin{abstract}
Artif Organs. 2000 May;24(5):346-54.
\end{abstract}
18. Feng Z, Nakamura T, Fujimoto T, Umezu M. In vitro investigation of opening behavior and hydrodynamics of bileaflet valves in the mitral position. Artif Organs. 2002 Jan;26(1):32-9. 
19. Förster F, Kaufmann R, Reul H, Rau G. A small pulsatile blood pump for

ventricular support during end-stage heart failure. Artif Organs. 2000 May;24(5):373-6.

20. Geier A, Kunert A, Albrecht G, Liebold A, Hoenicka M. Influence of Cannulation Site on Carotid Perfusion During Extracorporeal Membrane 22 Oxygenation in a Compliant Human Aortic Model. Ann Biomed Eng. 2017 Oct;45(10):2281-97.

21. Giridharan GA, Koenig SC, Kennington J, Sobieski MA, Chen J, Frankel SH, et 26

al. Performance evaluation of a pediatric viscous impeller pump for Fontan

cavopulmonary assist. J Thorac Cardiovasc Surg. 2013 Jan 1;145(1):249-57.

29 22. Gregory SD, Schummy E, Pearcy M, Pauls JP, Tansley G, Fraser JF, et al. A 30 Compliant, Banded Outflow Cannula for Decreased Afterload Sensitivity of 31 Rotary Right Ventricular Assist Devices. Artif Organs. 2015 Feb $1 ; 39(2): 102-9$.

32

33 23. Horvath D, Byram N, Karimov JH, Kuban B, Sunagawa G, Golding LAR, et al. ${ }^{34}$ Mechanism of SelfRegulation and In Vivo Performance of the Cleveland Clinic 35

36

37

38

39

40

41

the Hemodynamic Response Under Rotary Blood Pump 43

44

45

46

48

4

Artif Organs. 2000 Jan;24(1):57-63. 2017 Feb 17;12(2):e0172393.

Continuous-Flow Total Artificial Heart. Artif Organs. 2017 May;41(5):411-7.

24. König CS, Clark C. Flow mixing and fluid residence times in a model of a

ventricular assist device. Med Eng Phys. 2001 Mar;23(2):99-110.

25. Lim E, Dokos S, Salamonsen RF, Rosenfeldt FL, Ayre PJ, Lovell NH. Effect of 42

Parameter Variations on Assistance. Artif Organs. 2012 May 1;36(5):E125-37.

26. Mansouri M, Gregory SD, Salamonsen RF, Lovell NH, Stevens MC, Pauls JP, et al. Preload-based Starling-like control of rotary blood pumps: An in-vitro 47 evaluation. PLOS ONE.

27. Morsi YS, Sakhaeimanesh A, Clayton BR. Hydrodynamic evaluation of three 50 artificial aortic valve chambers. 
28. Ng BC, Kleinheyer M, Smith PA, Timms D, Cohn WE, Lim E. Pulsatile operation of a continuous-flow right ventricular assist device (RVAD) to improve vascular pulsatility. PLOS ONE. 2018 Apr 20;13(4):e0195975.

29. Ng BC, Smith PA, Nestler F, Timms D, Cohn WE, Lim E. Application of Adaptive Starling-Like Controller to Total Artificial Heart Using Dual Rotary Blood Pumps. Ann Biomed Eng. 2017 Mar 1;45(3):567-79.

30. Nishida M, Asztalos B, Yamane T, Masuzawa T, Tsukiya T, Endo S, et al. Flow 8 visualization study to improve hemocompatibility of a centrifugal blood pump. Artif Organs. 1999 Aug;23(8):697-703.

31. Noor MR, Ho CH, Parker KH, Simon AR, Banner NR, Bowles CT. Investigation of the Characteristics of HeartWare HVAD and Thoratec 13 HeartMate II Under Steady and Pulsatile Flow Conditions. Artif Organs. 2016

Jun 1;40(6):549-60.

32. Pauls JP, Stevens MC, Bartnikowski N, Fraser JF, Gregory SD, Tansley G. In Vitro Comparison of Active and Passive Physiological Control Systems for Biventricular Assist Devices. Ann Biomed Eng [Internet]. 2016 [cited 2018 May 21]; Available from: https://link.springer.com/article/10.1007\%2Fs10439-015$1425-1$

33. Pauls JP, Stevens MC, Bartnikowski N, Fraser JF, Gregory SD, Tansley G. 24 Evaluation of Physiological Control Systems for Rotary Left Ventricular Assist 25 Devices: an in vitro Study. Ann Biomed Eng. 2016 Aug 1;44(8):2377-87. 
27 34. Petrou A, Lee J, Dual S, Ochsner G, Meboldt M, Daners MS. Standardized 28

Comparison of Selected Physiological Controllers for Rotary Blood Pumps: In

29

30

31

32

33

34

35

36

38

Vitro Study. Artif Organs. 2018 Mar 1;42(3):E29-42.

35. Schumer E, Höffler K, Kuehn C, Slaughter M, Haverich A, Wiegmann B. Invitro evaluation of limitations and possibilities for the future use of intracorporeal gas exchangers placed in the upper lobe position. J Artif Organs. 2018 Mar 1;21(1):68-75.

36. Sénage T, Février D, Michel M, Pichot E, Duveau D, Tsui S, et al. A mock 37

circulatory system to assess the performance of continuous-flow left ventricular 39 assist devices (LVADs): does axial flow unload better than centrifugal LVAD? 40 ASAIO J Am Soc Artif Intern Organs 1992. 2014 Apr;60(2):140-7.

41

42 37. Stephens AF, Stevens MC, Gregory SD, Kleinheyer M, Salamonsen RF. In Vitro 43 Evaluation of an Immediate Response Starling-Like Controller for Dual Rotary 44 Blood Pumps. Artif Organs. 2017 Oct;41(10):911-22.

39. Burton DA, Stokes K, Hall GM. Physiological effects of exercise. Contin Educ Anaesth Crit Care Pain. 2004 Dec 1;4(6):185-8.

40. Guyton AC, Hall JE. Textbook of Medical Physiology. 11 edition. Philadelphia, Pa: Saunders; 2005. 1152 p.

Page 21 of 25

41. Levick RJ. An Introduction to Cardiovascular Physiology 5E. 5 edition. London: CRC Press; 2009. 432 p.

42. Korakianitis T, Shi Y. A concentrated parameter model for the human 7 cardiovascular system including heart valve dynamics and atrioventricular interaction. Med Eng Phys. 2006 Sep;28(7):613-28.

43. Kotchen TA. Developing Hypertension Guidelines: An Evolving Process. Am J Hypertens. 2014 Jun 1;27(6):765-72.

5

52

53

54

55 


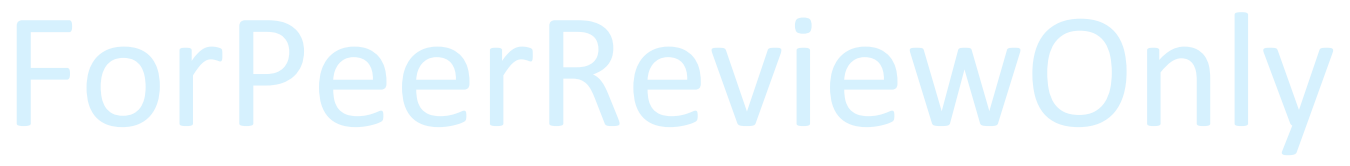

34

35

36

37

38

39

40

41

42

43

44

45

46

51

52

53

54

55

56

57

58

59

60

URL: http://mc.manuscriptcentral.com/tmet Email: John.Woodcock@CardiffandVale.wales.nhs.uk ${ }^{36}$ 
Table 1. Operating parameters for the MCTR design

\begin{tabular}{|l|l|}
\hline Heart rate $(\mathrm{bpm})$ & $30 \sim 180$ \\
\hline Ventricular pressure $(\mathrm{mmHg})$ & $0 \sim 200$ \\
\hline Atrial pressure $(\mathrm{mmHg})$ & $0 \sim 50$ \\
\hline Arterial pressure $(\mathrm{mmHg})$ & $30 \sim 200$ \\
\hline Cardiac output $(\mathrm{L} / \mathrm{min})$ & $2 \sim 18$ \\
\hline
\end{tabular}

Table 2. Specifications of the linear motors

\begin{tabular}{|c|c|c|c|}
\hline Force output $(\mathrm{N})$ & Stroke $(\mathrm{m})$ & $\begin{array}{c}\text { Maximum } \\
\text { velocity }(\mathrm{m} / \mathrm{s})\end{array}$ & $\begin{array}{c}\text { Maximum } \\
\text { acceleration }\left(\mathrm{m} / \mathrm{s}^{2}\right)\end{array}$ \\
\hline 200 & 0.1 & 3 & 150 \\
\hline
\end{tabular}


Page 23 of 25

1

2

51

52

53

54

55

56

57

58

59

60

URL: http://mc.manuscriptcentral.com/tmet Email: John.Woodcock@CardiffandVale.wales.nhs.uk ${ }^{39}$ 


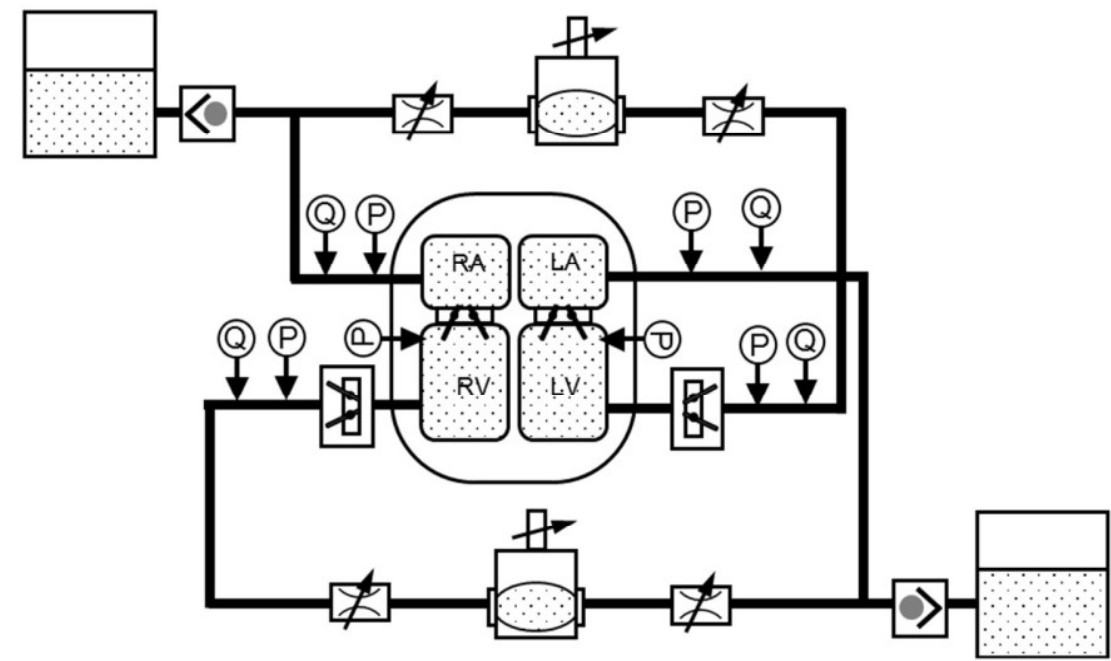

Throttle valve

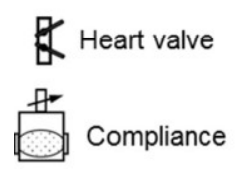

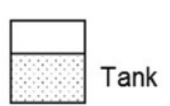

$D \quad$ One-way valve
(P) $\checkmark$ Pressure transducer

Q) Flow transducer

Fig. 1 System configuration of the mock circulatory test rig

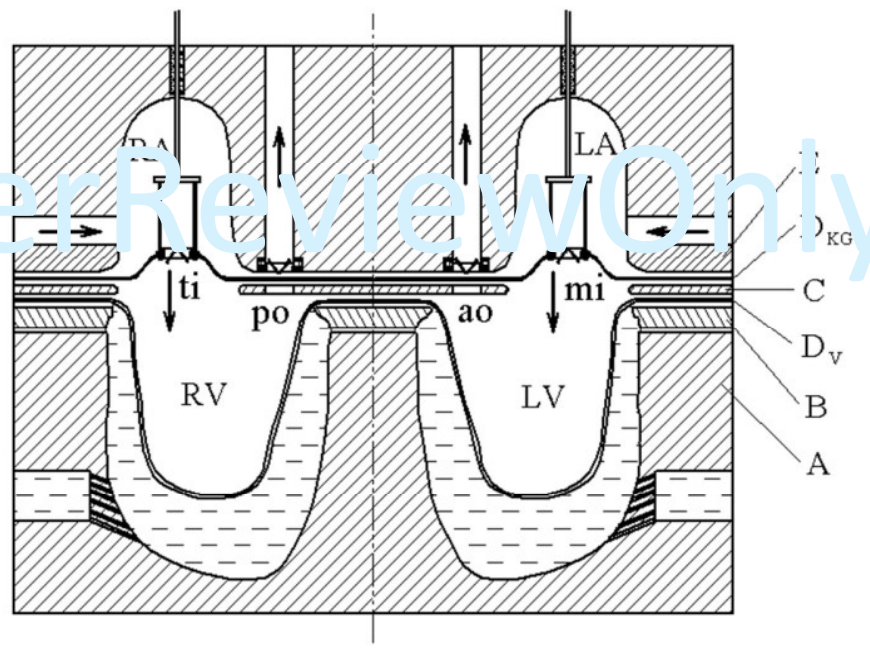

Fig. 2 Section view of the heart model in the test rig 
Page 24 of 25

URL: http://mc.manuscriptcentral.com/tmet Email: John.Woodcock@CardiffandVale.wales.nhs.uk ${ }^{42}$ 

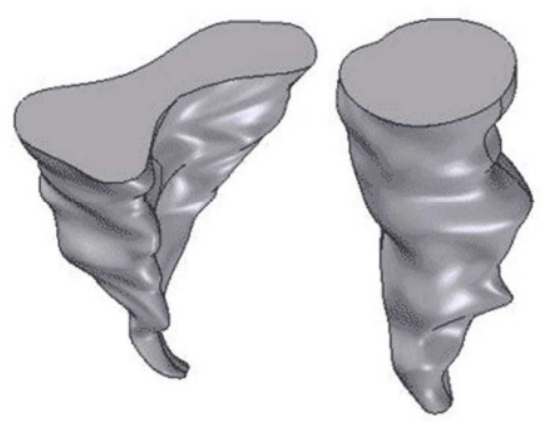

(a) End systolic phase
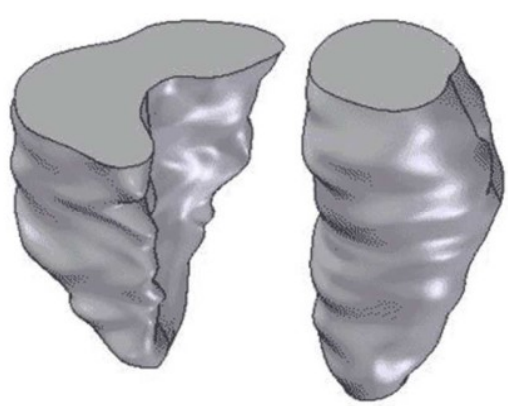

(b) End diastolic phase

Fig 3. Ventricle geometry reconstructed from MRI results

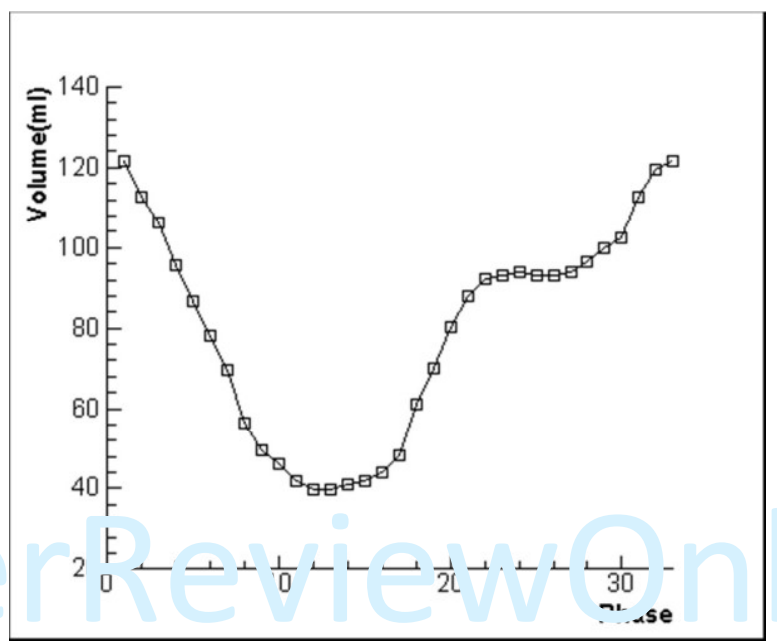

Fig. 4 Volume change of the left ventricle in a heart cycle measured with MRI

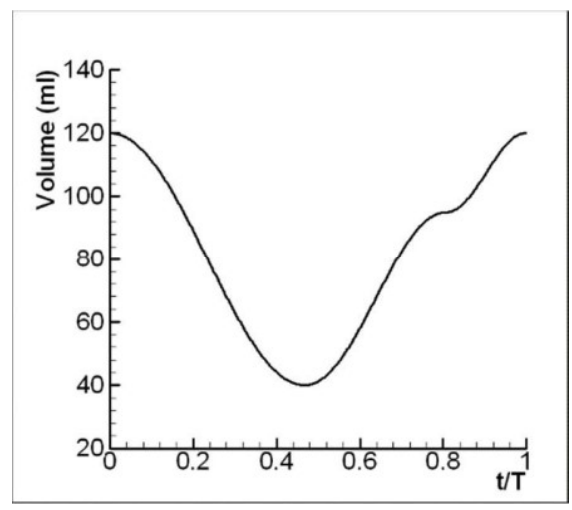


Fig. 5 Smoothed and approximated left ventricular volume changing 
Page 25 of 25

1

2

51

52

53

54

55

56

57

58

59

60

URL: http://mc.manuscriptcentral.com/tmet Email: John.Woodcock@CardiffandVale.wales.nhs.uk ${ }^{45}$ 


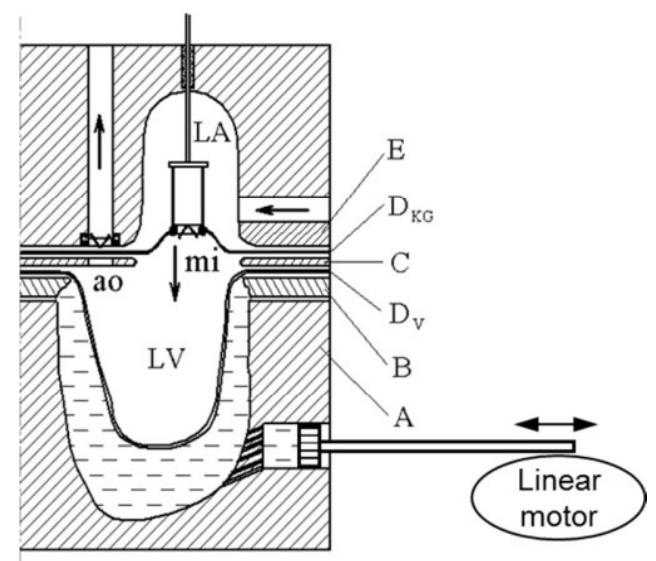

Fig. 6 Illustration of the ventricular motion actuated by the motor driven piston

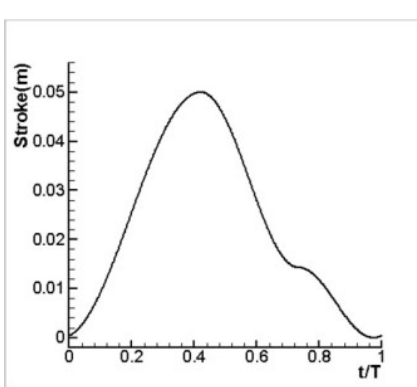

(a) Stroke

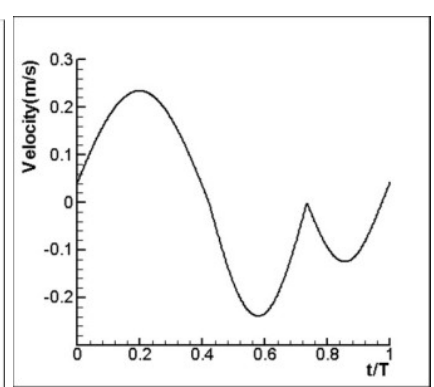

(b) Velocity

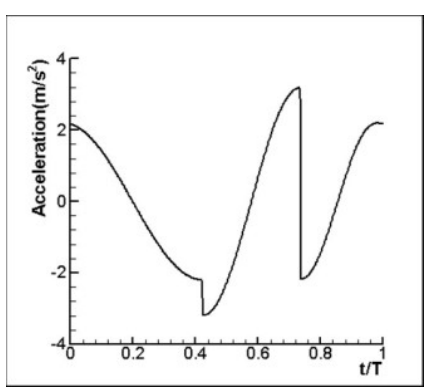

(c) Acceleration

Fig. 7 Motion profile of the ventricle actuating piston in an example of the rest condition in a healthy adult subject 
